Dominique Cardon Jean-Philippe Heurtin Anne-Sylvie Pharabod Sabine Rozier

\title{
Mais qui fait bouger le compteur du Téléthon ? Une construction télévisuelle de la solidarité
}

In: Sciences sociales et santé. Volume 16, n³, 1998. pp. 17-40.

Citer ce document / Cite this document :

Cardon Dominique, Heurtin Jean-Philippe, Pharabod Anne-Sylvie, Rozier Sabine. Mais qui fait bouger le compteur du Téléthon ? Une construction télévisuelle de la solidarité. In: Sciences sociales et santé. Volume 16, n³, 1998. pp. 17-40.

doi : $10.3406 /$ sosan.1998.1434

http://www.persee.fr/web/revues/home/prescript/article/sosan_0294-0337_1998_num_16_3_1434 


\section{Abstract}

Who makes shift the pledge meter of the French Telethon. A televisual construction of solidarity Since December of 1987, the Association française de lutte contre les myopathies (AFM) (the French Muscular Distrophy Association), organizes the French Telethon, in order to support scientific research and to help the victims of muscular diseases. Unlike most télévision programs, the Telethon is a unique apparatus which aim is to provoke solidarity, and as such is quite exemplary of the transformations that affect the televisual public sphère, the forms of the solidarity and the research public policies. This article deals with the role played by the pledge meter of the French Telethon, and analyses the conditions of emergence, during the show, of a bound up community. It tackles successively the television show, the reception of the program and the local events organized during the television program. The final figure of the meter can not be surely linked to the evolution, measured in real time, of the solidarity events; nor to the strategies of the producers of the program. That is by the variations of the figure, translating in accelerations and decelerations the different deals (monetary and human), which ties every one and ail to the courage of the sick persons and to the efforts of the scientists, that the meter device manage to corne into sight ail together the monetary value of the pledges and the solidarity between the people who make common cause in the Telethon community.

\section{Resumen}

Pero quién mueve el medidor del Téléthon ? La construcción televisual de la solidaridad Iniciado en 1987 por la Asociación Francesa contra las Miopatías (AFM) para recoger fondos con el fin de apoyar la investigación científica y la ayuda a los enfermos, el Téléthon constituye un dispositivo de solidaridad ejemplar de las transformaciones que afectan al mismo tiempo al espacio público televisual, al mundo de la solidaridad asociativa y a las políticas de orientación y de financiamiento de la investigación clínica. Este artículo, consagrado al papel que juega el medidor de promesas de donación del Téléthon, propone un análisis de las condiciones que encabezan la emergencia, durante la emisión, de una comunidad solidaria. Aborda sucesivamente la representación televisiva, la recepción del programa y todos los tipos de acciones locales que acompañan a la emisión. La evolución del medidor no puede ser solamente atribuida a las intenciones estratégicas de los programadores ni tampoco únicamente a la tarea de recuento estadistico del dinero recaudado. Es por su movimiento mismo, que traduce bajo la forma de aceleraciones o desaceleraciones las diferentes transacciones (monetarias y humanas) que ligan a todos y a cada uno con el valor de los enfermos y con los esfuerzos de los investigadores, que el dispositivo de recuento logra poner de relieve al mismo tiempo el valor monetario de las promesas de donación y la solidaridad entre las personas que se reconocen en la comunidad del Téléthon.

\section{Résumé}

Résumé. Initié en 1987 par l'Association française de lutte contre les myopathies (AFM) pour recueillir des fonds afin de soutenir la recherche scientifique et l'aide aux malades, le Téléthon constitue un dispositif de solidarisation exemplaire des transformations qui affectent tout à la fois l'espace public télévisuel, le monde de la solidarité associative et les politiques d'orientation et de financement de la recherche scientifique. Consacré au rôle joué par le compteur des promesses de don, cet article propose une analyse des conditions qui président à l'émergence, pendant l'émission, de la communauté solidaire du Téléthon. II aborde successivement la représentation télévisée, la réception du programme et les actions locales de toute nature qui entourent l'émission. L'évolution du compteur ne peut être imputée, ni aux seules intentions stratégiques des programmateurs, ni à la seule entreprise de représentation statistique de l'argent collecté. C'est par son mouvement même, en traduisant sous forme d'accélérations et de décélérations les différentes transactions (monétaires et humaines) qui lient tous et chacun au courage des malades et aux efforts des chercheurs, que le dispositif de comptage parvient à faire advenir ensemble la valeur monétaire des promesses de don et la solidarité des personnes qui s'unissent les unes aux autres dans la communauté du Téléthon.

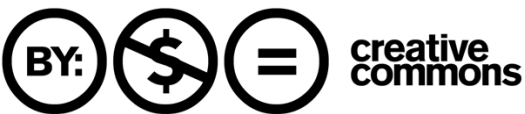


Sciences Sociales et Santé, Vol. 16, $n^{\circ} 3$, septembre 1998

\section{Mais qui fait bouger le compteur du Téléthon? Une construction télévisuelle de la solidarité}

Dominique Cardon*, Jean-Philippe Heurtin*, Anne-Sylvie Pharabod***, Sabine Rozier****

Résumé. Initié en 1987 par l'Association française de lutte contre les myopathies (AFM) pour recueillir des fonds afin de soutenir la recherche scientifique et l'aide aux malades, le Téléthon constitue un dispositif de solidarisation exemplaire des transformations qui affectent tout à la fois l'espace public télévisuel, le monde de la solidarité associative et les politiques d'orientation et de financement de la recherche scientifique. Consacré au rôle joué par le compteur des promesses de don, cet article propose une analyse des conditions qui président à l'émergence, pendant l'émission, de la communauté solidaire du Téléthon. Il aborde successi-

\footnotetext{
* Domique Cardon. sociologue. CNET. 38-40, rue du Général-Leclerc, 92131 Issy-lesMoulineaux Cedex, France.

** Jean-Philippe Heurtin, sociologue. GSPM/CNET, 38-40, rue du Général-Leclerc. 92131 Issy-les-Moulineaux Cedex. France.

*** Anne-Sylvic Pharabod, ethnologue, EHESS. 54, boulevard Raspail. 75006 Paris. France.

**** Sabine Rozier, Sciences politiques. Université Paris I. 17, rue de la Sorbonne. 75231 Paris Cedex 05. France.
} 
vement la représentation télévisée, la réception du programme et les actions locales de toute nature qui entourent l'émission. L'évolution du compteur ne peut être imputée, ni aux seules intentions stratégiques des programmateurs, ni à la seule entreprise de représentation statistique de l'argent collecté. C'est par son mouvement même, en traduisant sous forme d'accélérations et de décélérations les différentes transactions (monétaires et humaines) qui lient tous et chacun au courage des malades et aux efforts des chercheurs, que le dispositif de comptage parvient à faire advenir ensemble la valeur monétaire des promesses de don et la solidarité des personnes qui s'unissent les unes aux autres dans la communauté du Téléthon.

Mots clés : sociologie des médias, sociologie morale, Téléthon, télévision. sollicitation humanitaire, mobilisation collective, solidarité, don, engagement, émotions.

Qu'il soit du pouvoir de la télévision de faire exister à distance des communautés imaginées, qu'il soit de son ressort d'inviter le téléspectateur à se sentir partie prenante d'événements qu'elle a coproduits, que, même misanthrope ou ironique. le téléspectateur soit toujours appelé à la regarder avec d'autres, des autres qu'il ne connaît pas, qu'il ne connaîtra jamais, mais qu'il sait présents au même événement, que le spectacle télévisuel soit l'occasion de tant de discussions, de bavardages ou de railleries qu'elles en tissent un immense texte dont les ramifications deviennent vite inaccessibles à une quelconque totalisation, tout cela, depuis maintenant une quinzaine d'années, la sociologie de la télévision a entrepris de le montrer. Mieux que d'autres sans doute, Dayan et Katz (1996) dans leur travail sur la médiatisation des cérémonies nationalcs ont doté l'événement télévisuel du pouvoir de faire des communautés avec des outils de communication électronique, de susciter à domicile des engagements collectifs et de forger des solidarités entre anonymes (voir aussi Anderson, 1996 ; Meyrowitz, 1985). Si la sociologie des médias a longtemps fait polémiquer deux positions inconciliables, l'une attribuant à la télévision un pouvoir exagéré (en hypostasiant ses effets idéologiques), l'autre surestimant la capacité de résistance des téléspectateurs aux messages qui leur sont adressés, des travaux d'inspiration pragmatiste ont tenté d'apprécier la manière dont un même programme pouvait articuler différents régimes d'engagement, définissant à la fois les condi- 
tions de l'investissement du spectateur et celles de son retrait critique (Pharabod, 1993 ; Cardon, 1995). L'instrument télévisuel se voit alors doté du pouvoir d'établir entre les spectateurs des solidarités suffisamment fortes pour que la notion de "public " puisse endosser une signification autre que statistique et ne soit pas réduite à une agrégation d'individus atomisés ; mais il ne saurait pour autant être regardé comme une force capable de s'introduire dans la conscience du spectateur au point d'enfermer chacune de ses pensées, de ses attitudes ou de ses représentations dans un répertoire univoque (1). La mise au jour du pouvoir de la télévision n'interdit donc pas d'apprécier la richesse et la diversité des capacités interprétatives du public. Celui-ci s'exerce simplement par l'entremise de dispositifs circonscrits, dont les effets, canalisés et contenus, s'exercent dans un monde qui a aussi appris à relativiser et à critiquer les ressorts de l'instrument télévisuel (2).

S'ils ont contribué à restaurer la force des événements télévisuels qu'avaient dissoute certaines approches herméneutiques en ethnographie de la réception (voir Mattelart et Neveu, 1996), ces travaux laissent cependant une importante difficulté en suspens. Les phénomènes que cette ethnologie des mondes télévisuels - mariage audacieux de l'anthropologic culturelle et de la sémiologie des écrans - doit tenir ensemble, les gestes qu'il lui faut réunir, les actions qu'elle tente d'cmbrasser sont si disparates, dispersés et incommensurables qu'elle se trouve entravée par la faiblesse de son appareil probatoire. Pour combler la distance qui sépare le programme du public, elle doit soutenir des hypothèses audacieuses sur les opérations de symbolisation qui permettent la reconnaissance, par le spectateur, des instructions sémiotiques qui lui sont proposées. En maintenant une frontière étanche entre le média et le public, elle se voit contrainte de faire transiter leur commerce distant sur le terrain de l'imaginaire. Aussi est-il utile de réinscrire dans l'analyse les dispositifs matériels et les technologies de représentation qui permettent au programme et au public de se construire mutuellement (Hennion et Méadel, 1988). C'est

(1) Sur le développement d'une approche configurationnelle et plurielle des publics représentés dans les statistiques d'audience, voir Chalvon-Demersay et Rosental (1988).

(2) Comme le montrent les travaux de Pasquier (1995). les jeunes fans d'Hélène et les garçons savent tout à la fois investir les personnages de la série d'attributs « magiques". se laisser posséder par le jeu fictionnel, et s'en retirer dans une position d'extériorité qui emprunte la réserve ironique des détracteurs du feuilleton. 
précisément dans cette perspective que s'inscrit cette recherche collective portant sur le Téléthon (3).

\section{Une émission à compteur}

Initié en 1987 par l'Association française de lutte contre les myopathies (AFM) pour recueillir des fonds afin de soutenir la recherche scientifique et l'aide aux personnes atteintes de maladies neuro-musculaires, le Téléthon présente un dispositif de solidarisation exemplaire des transformations qui affectent tout à la fois l'espace public télévisuel, le monde de la solidarité associative et les politiques d'orientation et de financement de la recherche scientifique. Bien que ces différentes dimensions soient, pour partie, interdépendantes, on traitera ici simplement de la première en prenant pour guide le compteur des promesses de don qui constitue l'un des principaux personnages de l'émission (4).

En effet, le Téléthon se présente moins comme une émission de télévision que comme un dispositif de mobilisation. Depuis décembre 1987. l'AFM, France-Télévision et Radio-France sont parvenus à imposer un rendez-vous annuel à 1.3 million de donateurs et à plusieurs millions de

(3) Entreprise pendant le Téléthon 96. cette enquête collective a été menée dans des directions extrêmement différentes afin d'épouser, autant que possible, la forme réticulaire du dispositif de sollicitation. Cet article s'efforce de poser sommairement le cadre général d'une problématique dont les développements empiriques seront déployés dans des travaux à venir. Plusieurs chantiers ont été ouverts pour cette enquête : le suivi ethnographique du montage de l'opération avec les acteurs de l'AFM. de la télévision et les différents partenaires qui participent à la production de l'émission. une centaine d'entretiens téléphoniques avec des donateurs réalisés pendant le Téléthon, une analyse statistique du trafic téléphonique minuté des appels des donateurs, une enquête ethnographique auprès de soixante groupes de réception pendant le Téléthon et deux observations participantes des actions locales de la "Force $T$ ». Les quelques conclusions présentées dans ce texte s'appuient sur un premier dépouillement des matériaux recueillis sur ces différents terrains. Elles doivent donc être considérées comme de simples indications, susceptibles d'être contredites, précisées ou reformulées ultérieurement. Ce travail n'aurait pu être réalisé sans le soutien de l'AFM qui a accepté de nous ouvrir ses portes et de nous faire accéder au travail de préparation de l'émission.

(4) Concernant la politique scientifique de l'AFM qui tire une partie de sa spécificité de la contractualisation télévisuelle avec les donateurs, voir l'article de Callon et Rabcharisoa dans ce numéro. 
téléspectateurs et d'auditeurs. Trente heures durant. du vendredi soir au milieu de la nuit de samedi à dimanche, se succèdent devant les caméras des chercheurs et des chanteurs. des personnes malades et des sportifs, des chefs d'entreprise et des responsables d'association. des inconnus et des vedettes de télévision... À la différence de nombre de programmes de télévision (de variété, de talk-show ou d'information), l'émission ne s'organise pas autour d'un dispositif théâtral qui mettrait face-à-face une scène centrale et des spectateurs physiquement présents ou distants. La scène centrale, quasiment désertée par ses acteurs habituels, sc laisse progressivement conquérir par les participants que le programme a su mobiliser. À cet effet, le conducteur de l'émission orchestre, avec une régularité métronomique. des séquences qui tout au long de l'émission vont permettre au plateau central de se connecter à de multiples scènes locales. Tous les dispositifs permettant de rapprocher la télévision de son public sont ici mobilisés. Tantôt, celle-ci se transporte auprès de celui-là, au moyen de duplex télévisés avec des villes de province, de liaisons avec des journalistes de radios locales, ou de reportages transmis depuis les régions. Tantôt c'est le public qui vient à la télévision : ici c'est un donateur qui, en direct, converse au téléphone avec un animateur ; là, c'est la lettre d'un malade qui est lue à l'antenne. La présence du téléspectateur, même absent de l'image, est dès lors constamment activée au travers des diverses sollicitations - appels à téléphoner, à utiliser le Minitel ou Internet - dont il est la cible.

Le programme télévisé s'organise ainsi sous la forme d'un réseau étoilé. Après chaque connexion avec un point en périphérie, le programme revient toujours vers son centre organisateur dont les deux points de passage obligés sont l'animateur principal, Claude Sérillon. et le compteur des promesses de don. Une fois que le premier s'est tourné vers le second pour faire applaudir le nouveau chiffre des promesses de don. le programme repart vers un nouveau point du territoire télévisuel que maille le Téléthon. L'émission s'organise ainsi autour d'un plateau central, depuis lequel sont émis des messages de sensibilisation, qu'entoure une périphérie que le programme ne cesse d'aller visiter comme pour prendre la mesure des effets de la mobilisation qu'il a déclenchée. L'espace qui peut ainsi être parcouru se limite cependant aux différents nœuds du territoire français qui ont été équipés d'instruments télévisuels. Il se trouve donc limité par le budget du programme et les contraintes techniques de la télévision en multiduplex. Certes, les réseaux électroniques (le téléphone, le Minitel. Internet) permettent de s'immiscer jusque dans les foyers des téléspectateurs, mais ces derniers ne peuvent être directement représentés sous la forme d'une présence à l'écran. De sorte que. bien que principalement construite par et pour la télévision. la mobilisation du Téléthon 
déborde toujours les possibilités de sa couverture télévisuelle. Année après année, les responsables de l'AFM ont suscité et organisé des actions locales dont le déroulement s'est progressivement émancipé de l'émission, jusqu'à acquérir aujourd'hui une autonomie qui n'est pas loin de mettre en concurrence le programme télévisé et les actions festives de terrain qu'organise le réseau de bénévoles de l'AFM, la " Force $T$ ». Ces activités sont devenues si nombreuses (6 500 manifestations ont été dénombrées pour le Téléthon 96) que même les organisateurs de l'événement sont incapables de recenser tout ce que les gens font du et pour le Téléthon. Si la réalité sociale des pratiques solidaires effectuées pendant cet événement semble ainsi avoir échappé à sa couverture télévisuelle, il existe pourtant un outil de calcul qui parvient à mettre en perspective l'ensemble de l'opération, un instrument vers lequel convergent tous les mouvements, qui synchronise les actions et confère sa dynamique à l'ensemble de la mobilisation : le compteur des promesses de don.

Si le compteur peut être regardé comme un rouage essentiel de la coordination de la mobilisation, il peut aussi être considéré comme l'un des points les plus fragiles du Téléthon. Car en exhibant à l'image la progression chiffrée des promesses de don, il cristallise toutes les tensions qui traversent l'opération : il compte quand les autres donnent, il somme ce qui ne se compte pas, il calcule les gestes de gens qui disent ne pas calculer. Comment les organisateurs du Téléthon, qui font par ailleurs montre de tant d'ingéniosité et de savoir-faire, ont-ils pu livrer à leurs détracteurs un outil aussi facilement dénonçable ? Pourquoi avoir placé l'horizon solidaire de la générosité collective sous le signe d'un calculateur ? Le compteur focalise le débat qui, dans le langage moral ordinaire comme dans le discours des sciences humaines, oppose de façon intransigeante l'artificialité des procédures télévisuelles du marketing humanitaire et l'authenticité des mouvements de solidarité collective.

Enfermer le Téléthon dans cette polémique n'apparaît guère pertinent dès lors que l'on examine attentivement les opérations au travers desquelles il prend forme. Le dispositif permet, en effet, aux individus et aux groupes qui se solidarisent de se manifester mutuellement les liens qui les unissent au-delà du programme télévisé. De sorte que l'évolution du compteur ne peut être imputée ni aux seuls calculs stratégiques des programmateurs ni à la seule entreprise de représentation statistique de l'argent collecté. C'est par son mouvement même, en traduisant sous forme d'accélérations et de décélérations les différentes transactions (monétaires et humaines) qui lient tous et chacun au courage des malades et aux efforts des chercheurs, que le dispositif de comptage parvient à faire advenir ensemble la valeur monétaire des promesses de don et la solidarité entre les personnes qui s'unissent les unes aux autres dans la communauté du 
Téléthon. Il s'agira donc moins ici d'opposer l'arbitraire à l'authenticité, l'intérêt au désintérêt, la gratuité au calcul, la spontanéité à la contrainte que de montrer comment la manifestation d'un être collectif aussi imposant que les treize cent mille donateurs du Téléthon doit une partie de sa logique aux transformations que l'incessant mouvement du compteur imprime au programme, aux téléspectateurs et aux personnes engagées dans les actions locales.

\section{Le compteur en mouvement}

Arrêtons-nous, en premier lieu, sur le statut complexe du compteur au sein de la représentation télévisuelle. Celui-ci apparaît à la fois comme un outil mesurant les dons et comme un instrument destiné à les accélérer. Les chiffres du compteur réferent à la fois à un acteur extérieur au programme (le public) et à un agent interne (les programmateurs). Et c'est, pour une part, le déplacement élastique et incessant entre l'extérieur et l'intérieur du programme, entre indifférence et sensibilisation, critique et engagement, que le mouvement imprévisible du compteur du Téléthon permet d'entretenir tout au long du programme. En effet, tels qu'ils se donnent immédiatement à lire pour le téléspectateur, les termes du contrat de participation qui l'impliquent sont simples et ne présentent pas d'équivoque. L'instrument compteur est interpellé par les animateurs sur le mode du constat. Comme tous les outils de mesure animés, il « monte »; " grimpe » ou " se bloque ». Son indépendance à l'égard des actions des programmateurs est mise en scène avec insistance. Pour le consulter, il faut le rejoindre, se tourner vers lui, prélever une mesure et établir un constat : "Au compteur il est 22 heures. 249 millions 284 milles » (Claude Sérillon, Téléthon 96, samedi, 21:59); "Allez en avant, on lève la tête, on regarde ce compteur qui tourne et qui continue de tourner, gentiment au début, je pense que ce n'est évidemment pas encore assez mais qu'il y a encore beaucoup de gens dans les rues » (Gérard Holtz, Téléthon 1995, vendredi, 19:53). Pendant toute la durée du programme, les animateurs multiplient les formules en direction des téléspectateurs qui associent directement le geste individuel du don et la sommation collective des générosités. "Vous appelez le 36.37 ", demande par exemple Gérard Holtz, "et lorsque vous appelez le 36.37, vous faites bouger le compteur à vue d'oeil » (Téléthon 94). Le rythme soutenu de ses évolutions, un changement toutes les deux ou trois minutes, vise premièrement à le faire apparaître comme directement connecté à l'évolution mesurée en temps réel des actions de solidarité accomplies en direction du programme et 
secondairement à donner au public le spectacle ininterrompu de cette mesure. Deux présupposés voisins sont essentiels à cette identification " réaliste » du chiffre du compteur : il parvient à reconnaître et à représenter un état du monde réel, les donateurs, et ce monde dispose d'une autonomie relative à l'égard de la télévision. Il existe alors comme un écart, une distance entre programmateurs et public qui autorise les sollicitations des premiers à exercer des effets sur les actions du second et permet au compteur d'être le témoin fidèle et l'instrument de mesure de leurs échanges. Le compteur doit ici respecter une très forte exigence de fidélité. S'il est bien l'instrument qui représente, à l'intérieur du programme, ceux qui se trouvent à l'extérieur, alors chacun des nouveaux donateurs doit pouvoir voir son don s'afficher sur l'écran. Affichant au franc près la sommation des promesses, révisant constamment l'ancien montant par un nouveau, le compteur s'apparente à une succession de mises au point photographiques, sans cesse réajustées en fonction de l'évolution des gestes de dons.

Cependant, le compteur est aussi interpellé par les animateurs sur un mode qui relève moins du constat que de celui de la performance. On va lui parler, le faire agir, lui demander de répondre, de ne pas trahir et de continuer à fédérer les énergies. Il faut « le réveiller », " le faire bouger», lui faire « dépasser ses limites » ct " sauter les chiffres », " ne pas le laisser se refroidir » ou "s'endormir ». Tous - animateurs, téléspectateurs, acteurs locaux - multiplient leurs encouragements et leurs exhortations afin de tenter de lui imprimer leur force. Dès que le programme s'enflamme, que les animateurs, le public et les participants font montre d'enthousiasme. le point d'inertic du compteur se déplace de l'extérieur vers l'intérieur du programme. Les acteurs du plateau central se dotent mutuellement du pouvoir d'accélérer eux-mêmes son mouvement : "Le compteur est à presque à 16 millions de francs. Ce n'est qu'un tout petit début, Serge Lama on compte sur vous comme parrain pour accélérer le tout » (Claude Sćrillon, Téléthon 95, vendredi, 21:10). Ou encore, pendant le Téléthon 96 : "Thierry Lhermitte a fait exploser le Minitel, il a fait exploser Internet. On est à 250 millions » (Claude Sérillon, samedi, 22:10). Le compteur perd alors sa fonction de sommation pour s'en voir assigner une autre, performative cette fois, celle consistant à susciter chez les téléspectateurs le désir de le voir accélérer son mouvement. Il ne reflète plus, il fait agir. D'instrument de mesure, il se mue en instrument servant à agir sur la mesure. Le chiffre du compteur ne renvoie dès lors plus à une extériorité plus ou moins bien " représentée » dans le programme, mais aux stratégies internes déployées par les programmateurs pour rendre présent un public de chiffres lumineux. Les chiffres du compteur ne réfèrent plus à un état du monde, mais à l'ensemble des anticipations et des calculs, des diverses technologies rhétoriques, inspirées du marketing de la sensibili- 
sation humanitaire, et destinées à emporter l'adhésion du téléspectateur. Le compteur avance pas à pas, puis s'accélère d'un coup, se bloque, pour mieux repartir lorsqu'un reportage vient de s'achever. Dès que l'animateur se tourne vers lui, il s'ébroue avec plus d'insistance qu'à l'accoutumé. Lorsqu'une unité supéricure s'annonce («le mur des 200 millions»), le passage fait l'objet d'une mise en scène spécifique. Plus encore, le compteur est invoqué dans tout un ensemble de stratégies persuasives. Le chiffre est mis en compétition avec sa performance de l'année précédente : "C'est la première fois qu'à cette heure de la nuit on passe les 60 millions " (Gérard Holtz, Téléthon 94, samedi, 00:18) ; des objectifs intermédiaires lui sont assignés : "Deux cent millions, 200 millions pour 20 heures, hein! je veux absolument 200 millions pour 20 heures moi. C'est mon pari personnel. Il le faut! Voilà » (Serge Lama, Téléthon 95, samedi, 18:49) ; les compteurs régionaux sont mis en concurrence : "C'est l'Hérault qui donne le plus, il faut que les autres départements se réveillent » (Téléthon 94, vendredi, 19:50) ; les groupes sociaux sont mis en compétition : "Dix francs, c'est pas un petit don. Ce sont les personnes âgés qui appellent. Que font les jeunes? " (Valérie Maurice, une animatrice, Téléthon 94, samedi, 14:25), etc. Une des particularités de l'émission tient au fait que cette mise en scène télévisuelle du compteur n'est pas dissimulée aux téléspectateurs. Parfois même, l'artificialité du procédé de mise en mouvement du compteur est montrée de façon ostensible à l'écran. En 1996. Thierry Lhermitte, le parrain de l'ćmission, utilisera tout au long de l'émission une baguette magique pour faire avancer les chiffres. En 1995, à Maureen Door qui s'étonne : "C'est génial quand inême! À chaque fois que vous dites le chiffre, ça change!", Claude Sérillon n'hésite pas à confesser : "Oui je crois que je dois comnaître des gens derrière, c'est plutôt ça!».

Á l'écran, le point de référence du compteur oscille ainsi, sans être inquiété, entre réalisme et artificialisme. Cette variation est d'abord la conséquence du travail entrepris par les membres de l'AFM et les professionnels de télévision pour, en coulisse, le faire fonctionner. Sans explorer ici le dispositif technique mis en place par l'AFM, indiquons simplement que les acteurs du Téléthon réalisent un croisement continu de deux techniques radicalement différentes de production du chiffre. Toutes les demi-heures, un chiffre "réel " fournit une représentation des promesses de dons aux acteurs de l'AFM, correspondant à la demi-heure précédente. Il est calculé à l'aide d'un système complexe de capteurs placés dans les centres de promesses téléphoniques et sur le terrain de la «Force $T$ », afin de faire remonter, grâce à une chaîne de bénévoles, le montant des promesses enregistrées. Cette mesure cadre les possibles, fixe des bornes précises aux animateurs du chiffre. Elle donne l'angle de la pente. 
un point de départ et une estimation du point d'arrivée. À partir de cet étalon, une vie va pouvoir être donnée au compteur pour que d'instrument de représentation, il se mue en acteur de la représentation, en un performeur, capable par ses actions de faire agir les autres sur son état. Le compteur partage donc deux corps en référence : le public et les programmateurs. Mais, dans ce partage, le compteur échappe aux logiques exclusives de ces deux corps : en brouillant sa référence, il devient ainsi imprévisible. En s'affranchissant de ces deux logiques, sa référence finit par s'indexer sur son propre mouvement. Et son rôle au sein de la représentation se modifie selon la variation de son chiffre. Ainsi, l'émission réserve certaines séquences à une simple exposition du compteur, comme instrument d'appréciation d'un état (notamment, lors des moments calmes et routiniers du programme), ou comme instrument d'enregistrement d'un écart (notamment dans les moments de spectacularisation de la sollicitation). Ces glissements de rôles du compteur sont étroitement liés à la programmation alternante des différentes séquences du Téléthon : en faisảnt se succéder reportages, chansons, explications scientifiques et animations locales, le programme propose au téléspectateur des moments graves et des moments distrayants, des séquences fortes et des séquences faibles, des images attractives et des séquences de moindre intérêt (ce qui permet notamment au téléspectateur de quitter le programme pour téléphoner). La courbe des appels téléphoniques au 36-37 témoigne, par une succession de saccades, de pics et de creux oscillant minute après minute avec une très grande amplitude. de cette alternance du programme qui, pour éviter de retenir le téléspectateur dans la gravité et le sérieux, aménage des moments de repli, de distanciation et d'ironie. Cette variation du programme réserve alors au téléspectateur la possibilité tour à tour de prendre le compteur au sérieux et d'en déconstruire les artifices, d'accepter sa référence comme réaliste et d'y lire les effets d'une mise en scène performative.

En effet, si on s'attarde sur les comptes rendus des discussions des téléspectateurs devant le programme, il est frappant de voir à quel point les plus engagés dans la cause décryptent aisément les stratégies déployées par les programmateurs (Liebes et Katz, 1993). Cependant, la capacité à décoder la machinerie télévisuelle du Téléthon n'interdit en rien de se reconnaître et de s'assumer comme donateur. L'engagement dans le Téléthon oppose donc moins les personnes selon leur socialisation ou leurs capacités cognitives (dont la distribution inégale contribuerait à cliver différents publics) que des grammaires interprétatives activées séquentiellement par les mêmes personnes. Ainsi, il n'est pas besoin d'être hostile au Téléthon pour reconnaître la place du compteur dans un scénario destiné à attacher les téléspectateurs au programme : "C'est un peu comme un pari. On se demande jusqu'où ça va aller"; "C'est comme 
une compétition pour savoir qui dit mieux. "Cependant, selon un procédé de détournement assez classique, si les téléspectateurs reconnaissent bien la dimension instrumentale du compteur, ils considèrent aussi qu'ils ne peuvent en être les victimes (ils ont « vu le truc »). À les entendre, sa force performative ne s'exercerait que sur d'autres, moins lucides ou moins clairvoyants qu'eux : «Je pense que c'est nécessaire, mêrme si ça peut paraître un peu futile, c'est nécessaire parce que ça donne effectivement une sorte d'émulation, une dynamique, c'est ça. Les gens vont dire: "On va essayer d'aller plus, on va essayer d'aller plus..." "; "Ça donne envie aux gens de remplir ce panier un peu plus. Peut-être, ça incite "; "Moi, je pense que c'est vachement important, ça tient les gens graves!' (Compte rendu de réception $\left.\mathrm{n}^{\circ} \mathrm{A}\right)$. La réception du Téléthon renoue bien ainsi avec la formule que Mannoni (1977) avait mise en exergue dans son travail sur l'imaginaire (voir aussi Favret-Saada, 1977). En effet, les télespectateurs disent : "Je vois bien que c'est un dispositif qui performe le don, mais quand même ", si bien que la question qui doit être posée aux téléspectateurs capables de procéder, parfois dans un délai très bref, à des engagements et des dégagements est de savoir quel est le principe qui leur permet d'arbitrer en faveur de l'action plutôt que de l'indifférence.

\section{La force des malades comme modèle moral d'engagement}

Cette question invite à explorer les raisons de l'investissement des personnes dans le Téléthon. Ouverte depuis la position du spectateur, l'émissión semble n'offrir d'emblée que deux propositions d'engagement : celle que configure une grammaire de la pitié à l'endroit des malades représentés et celle, inverse, de la critique et du refus de cette forme de sensibilisation aux malheurs des malades (5). Le téléspectateur

(5) La grammaire de la pitié s'articule à une situation caractérisée par l'existence de deux classes d'hommes inégaux sous le rapport du bonheur et par la possibilité d'un contact à distance entre des malheureux et des spectateurs qui, eux, ne souffrent pas. Le concernement sympathique aux malades s'accomplit par le biais de l'imagination. Celle-ci permet de surmonter la distance et d'expérimenter des changements de places ; le spectateur cherche ainsi à imaginer ce qu'il éprouverait à la place du malheureux. Dans le dispositif du Téléthon, où la question de la recherche d'un auteur du malheur (ici, de la maladie) ne se pose pas, le concernement sympathique s'accomplit dans la relation bienfaisante aux malades et le transport imaginatif à la place des malades se coordonne avec les sentiments de gratitude que l'intervention des bienfaiteurs leur inspire (Boltanski, 1993). 
n'aurait au fond le choix qu'entre deux positions exclusives l'une de l'autre : soit l'attendrissement soudain envers la souffrance des jeunes myopathes, et la sympathie avec les sentiments de gratitude que l'intervention des bienfaiteurs lui inspire; soit la distanciation, et la restauration des intentions derrière le surgissement des émotions sympathiques. Sans vouloir nier que l'imagination sympathique puisse jouer - ni qu'elle joue effectivement - dans la réception du Téléthon, nous voudrions avancer que le Téléthon offre aussi une autre proposition d'engagement. Deux dimensions de l'émission viennent, en effet, perturber une compréhension de son principe depuis la scule grammaire de la pitié. En effet, il apparaît clairement que ce qui distingue en premier lieu la sollicitation qu' organise le Téléthon, c'est bien d'abord son aspect ludique, joyeux et festif, presque chahuteur, le plus souvent occupé à représenter des personnes à se dépenser physiquement. C'est bien ce que P. Bruckner pointait, dans les quelques pages critiques consacrées au Téléthon de son livre La tentation de l'innocence (1995) : "On s'excusera de rappeler que la charité ne peut être amusante, qu'elle doit être "un peu sévère" (...) au risque de dégénérer en plaisanterie " (p. 249) et surtout à propos de cette frénésie d'action qui semble animer les participants du spectacle : "En fait cette démangeaison de mobilité est une sorte de vérification par l'absurde : plus les malades sont impotents, plus les bienfaiteurs gambadent, courent, grimpent, pédalent comme s'ils voulaient s'assurer de leur parfaite santé. " La seconde dimension du Téléthon, qui l'éloigne de la grammaire canonique du concernement sympathique face à la souffrance, relève précisément de la construction très particulière que l'émission effectue de l'image des malades. Ceux-ci, saisis dans les petits reportages qui jalonnent le Téléthon, sont, de manière presque systématique, pris dans un récit qui, à la manière d'un conte moral, retrace un "retour à la vie ", et dote une nouvelle fois les sujets de l'ensemble des attributs de l'humanité. Si les premières séquences sont toujours le rappel du malheur, la manifestation de la souffrance et de la difficulté à vivre dans le monde des bicn portants, la suite des reportages montre toujours la façon dont, à force de courage, de ténacité, de force intérieure, les malades surmontent leurs incapacités physiques. Après la première qualification des débuts de reportage. le fil narratif redéploie l'infini des potentialités qui, malgré le handicap, demeure.

Dès lors, ce que le téléspectateur peut découvrir à l'écran, c'est que, bien sûr, les malades du Téléthon sont faibles, mais qu'ils sont aussi forts (ils ont du courage, ils ont su faire de leur faiblesse un exemple de courage). C'est dire que le malade à la télévision est amoindri du fait de sa maladie - son corps est toujours en deçà de son vouloir -, mais du fait de son comportement, de sa beauté, de sa voix, de son courage, de l'éner- 
gie qu'il met à faire ce qu'il fait, on peut le considérer comme doué d'une force. Le téléspectateur, à l'inverse, regardant la télévision est bien portant. Mais, dans sa vie de tous les jours fait-il montre d'une même énergie que le malade qu'il voit ? Certainement non et c'est bien ce que lui montre le malade à la télévision : le spectateur se révèle à lui-même qu'il a des forces qui ne sont pas employées, des négativités à conjurer (égoïsme, indifférence, paresse, conflits d'intérêts, etc.). Le malade est souffrant mais plein de force et de courage, alors que le téléspectateur est bien portant, mais ne fait pas assez usage de cette bonne santé, la dilapide : «C'est vrai que c'est des leçons de courage, hein. Nous nous plaignons pour un oui, pour un non " (groupe réception $n^{\circ} \mathrm{D}$ ). Remarquable ainsi est la façon dont ces comptes rendus recomposent l'asymétrie de la rclation entre malades et spectateurs. Dans le cas de la pitié, la relation du spectateur au malheureux réserve la plénitude de la puissance d'action au premier et emporte avec elle une obligation morale d'engagement, ne serait-il qu'émotionnel. Dans une configuration marquée par la reconnaissance de la faiblesse du téléspectateur, l'asymétrie de la relation s'inverse et tend à faire du malheureux un exemple. Le téléspectateur est invité à reconnaître la leçon que lui adressent les malades, leur courage et à montrer, à son tour, le meilleur de lui-même et. afin d'être " à leur hauteur ». de faire triompher en soi la " positivité » sur la " négativité ». C'est ce qu'en substance affirmait J.-C. Petit, journaliste à La Vie, au cours du Téléthon 1996 : "Je trouve que le Téléthon, c'est une manifestation de la société française qui ne va pas bien, qui est crispée, qui est un petit peu en crise dans cette fin de siècle, et nous le sentons tous, et que le Téléthon, c'est le moment où nous sommes tous appelés à la pertinence et à l'excellence, à nous élever. Et je trouve ça formidable " (Téléthon, samedi, 00:27). Ce faisant, en suivant leur exemple, le téléspectatcur montre que tous appartiennent à une même humanité.

Comment, pour autant, le téléspectateur peut-il manifester ce lien au malade, cet enracinement dans une même communauté des existences humaines ? La réponse du Télćthon est claire et elle est continuellement répétée tout au long des trente heures d'émission : en agissant. Il ne s'agit pas simplement de parler, de manifester verbalement son émotion. L'exigence que déploie le Téléthon est d'agir sur soi-même et, plus simplement encore, d'agir. On saisit bien alors dans quelle mesure ce modèle tend à faire disparaître le spectateur, ou du moins à en destituer la position, en tant qu'elle est passive. La leçon donnée par les malades de nourrir en soi la vie, manifeste au spectateur une vocation en tant qu'être humain ; vocation qui est à étendre et à manifester à son tour. Dès lors. les actions entreprises à l'exemple des malades deviennent à leur tour des exemples pour tous les autres. Cette proposition d'engagement indique donc bien que 
'l'action, la dépense d'énergie physique, n'est pas tant destinée à assurer le spectateur de sa « parfaite santé », comme le disait Bruckner, mais à manifester le lien qui l'unit à l'ensemble des acteurs du Téléthon : l'action est le vecteur même de la construction de la communauté solidaire des donateurs, des téléspectateurs, des chercheurs et des malades.

\section{Les dispositifs locaux de solidarisation}

Agir en public avec d'autres, c'est bien là le trait commun à toutes les manifestations organisées sur tout le territoire national à l'occasion du Téléthon. Depuis que ce dispositif de sollicitation a été importé en France, en 1987, le nombre d'actions locales organisées en son nom n'a cessé de grossir au point que, dix ans après sa création, près d'un tiers de la somme récoltée provient de ce que les organisateurs appellent la « Force T». Lancers de petits pois, défilés de rois mages, courses de brouettes : c'est moins le caractère incongru ou spectaculaire des actions proposées qui appellera ici l'attention que le type de lien, à la fois moral et social, qu'elles contribuent à tracer entre tous ceux qui se mobilisent à cette occasion. Car le propre du Téléthon réside moins dans le caractère novateur de la mobilisation qu'il suscite - il ne fait en effet que réactiver des formes d'actions préexistantes - que dans l'intrication ingénieuse d'un dispositif de solidarisation festif et d'un dispositif de collecte de dons qui viendra nourrir le compteur du plateau central.

La « Force T $\mathrm{T}$, en effet, n'a pas l'apanage de l'exubérance et des débordements. La participation active du spectateur à l'objet du divertissement, l'exhibition du public en objet de la représentation, l'orchestration de la simultanéité festive, l'attachement à la commensalité, l'activation d'allégories fraternelles, la dramaturgie de la communion des cours, la célébration de la diversité dans l'unité, la conjugaison d'un patriotisme de clocher et d'une solidarité nationale, tout cela, le Téléthon est loin de l'avoir inventé. Aussi est-il nécessaire de ne pas surestimer le caractère novateur de la " Force $\mathrm{T}$ » qui s'apparente surtout à une réappropriation de pratiques locales, festives ou de bienfaisance, aménagées de façon à s'inscrire dans le dispositif de mobilisation télévisuel. La seule exigence que les responsables du Téléthon imposent aux organisateurs de ces manifestations locales réside dans l'incessant travail de mesure et de dénombrement des liens de solidarité auxquels elles donnent lieu. En effet, l'observateur ne peut être que frappé, tant à l'antenne, lorsque sont retransmises des images de la « Force $T$ », que dans les arènes soustraites aux caméras, par l'abondante prolifération de chiffres que le Téléthon sus- 
cite. Ce ne sont que comptes et décomptes, sommations (de kilomètres, de longueurs d'échelle, de litres de lait, de trombones, etc.), comparaisons, surenchères et records. Ces chiffres sont régulièrement exhibés et commentés et les participants constamment exhortés à les dépasser. Ils témoignent, à leur manière, des principales caractéristiques des dispositifs d'engagements dans la « Force $T$ ». Leur particularité est de ne pouvoir faire l'objet d'aucune appropriation individuelle - car ils incarnent le produit d'un effort collectif, ouvert à tous, et que personne, individuellement, ne peut revendiquer - et de mesurer des biens qui ne peuvent faire l'objet d'aucune marchandisation en dehors du Téléthon. Ne mesurant, ni la force d'une individualité, ni la valeur d'un produit, les chiffres du Téléthon dénombrent des gestes, des actions et des liens entre personnes.

Chaque manifestation de la « Force $T$ » se voit adjoindre un dispositif métrologique visant à découper les actions en séquences homogènes afin de produire une valeur discrète achetée par les spectateurs (qui, bien souvent, sont de futurs acteurs) et de transformer ainsi chaque valeurgeste en valeur-argent. Seules les manifestations à caractère théâtral, organisant un face-à-face entre des acteurs et un public sont dispensées d'un tel travail de découpage et se voient associer un dispositif extérieur de collecte (par exemple, une urne dans laquelle sont déposées des promesses de don), de sorte que le compteur, cet instrument vers lequel convergent toutes les branches du réseau étoilé de la « Force $T$ », en vient à agréger la représentation chiffrée de gestes très disparates (des coups de pédale, des confections de mailles, etc.), a priori incommensurables et irréductibles les uns aux autres.

Dans un tel dispositif, action et solidarisation vont de pair. Aussi estil aisément compréhensible que le degré d'ouverture des manifestations au public soit maximum. Le coût d'entrée y est bien inférieur à celui qu'exigent d'autres formes de mobilisation, notamment les plus militantes. Quiconque le désire peut en effet aisément trouver, dans l'infinie variété des manifestations localcs de la « Force $T$ ", une offre de participation ajustée à ses possibilités et ce, quels que soient son âge, son sexe, ses compétences et sa condition physique. Les participants sont toutefois soumis à des règles du jeu particulières (descriptibles sous la forme de " défis »), qui contribuent à transformer la signification et l'usage de leurs gestes, tout en valorisant les qualités et les compétences sur lesquels ils s'adossent. Les pâtissiers sont certes invités à faire des gâteaux, les cyclistes à pédaler, les militaires à exhiber leurs engins motorisés, les danscurs à danser, les grands-mères à tricoter, etc., mais l'énergie que les uns et les autres déploient pour faire ce qu'ils ont l'habitude de faire - parce qu'il s'agit là de leur métier, d'une activité de loisir, d'un tour de main se voit soumise à des contraintes temporelles inédites, à des exigences de 
performance, à une obligation d'agir en public (sous le regard des autres), et/ou à la nécessité de se coordonner avec les autres : le pâtissier contribuera à la confection d'un gigantesque pithiviers de plusieurs mètres de long, l'amatcur de courses cyclistes sera chargé de nourrir de coups de pédales, des heures durant, la puissante dynamo de son vélo afin d'alimenter en énergie l'ampoule éclairant les huit lettres du mot « Téléthon » situées au sommet du kiosque à musique,; les militaires désosseront puis remonteront une automobile tout terrain, les danseurs se rclaieront des heures durant pour ne pas rompre la « danse de 30 heures », les grandsmères s'emploieront à tricoter une immense écharpe destinée à ceindre le clocher, etc. Chaque participant, en "donnant de lui-même » et en y " mettant du sien ", comme l'on dit, contribue à incorporer quelque chose de lui-même dans l'action en train de se faire et à inscrire dans son geste " une parcelle de sa nature et de sa substance » (Mauss, 1950:161). La « Force $T$ » offre ainsi aux individus la possibilité de faire un don chargé de leur personne et ce, de manière peut-être plus intense et plus durable que pour un don d'argent, qui, bien qu'il ne puisse s'effectuer qu'au travers d'actes concrets - téléphoner, promettre, signer un chèque, retourner l'enveloppe - peut toujours être regardé comme un geste moins " engageant » qu'une participation à une manifestation collective et publique, en raison de son caractère relativement anonyme et silencieux.

Les actions locales du Téléthon enferment ainsi une tension difficile à surmonter entre le singulier et le commun : chaque participant y est en effet moins regardé comme un être doté de qualités particulières (ancien combattant, membre de la paroisse, danseur de flamenco, etc.) que comme un individu surtout caractérisable par la possession d'attributs communs partagés avec d'autres. La variété sans la différence, la valorisation sans l'ostentation, la personnalisation sans l'individualisation : ce sont là quelques-unes des gageures auxquelles doivent faire face les animateurs locaux du Téléthon. L'idéal égalitariste qu'enferme cet événement appelle également une absence relative de distinction des rôles assumés par les participants, en ce sens que ces derniers sont constamment invités à glisser d'une place à une autre, à ne pas s'enfermer dans une posture, un geste ou une activité particulière mais à saisir les différentes prises et les diverses opportunités d'engagement adressées par les collectifs en mouvement qu'ils sont amenés à traverser. La circularité et l'interchangeabilité que favorise une telle configuration de places préserve indéniablement du ridicule ceux qui y sont " pris ». Elle rend également difficile le maintien des spectateurs dans une position d'extériorité. Toute situation insulaire tendant à tracer une frontière invisible entre ceux qui agissent et ceux qui regardent est en effet lourde de menace car elle favorise la distanciation, l'ironie et la critique. Aussi, les actions du Téléthon qui tendent à ins- 
taurer une séparation étanche entre un public et des acteurs (comme les scènes à caractère théâtral ou les cortèges linéaires) sont-elles conçues de façon à favoriser une circulation entre la scène et le public (en invitant un spectateur à prendre part au jeu, en faisant déborder l'action au sein du public), ou à enrôler topographiquement le corps des spectateurs dans le cercle que dessine un cortège (entourant une place de village plutôt que de le traverser de part en part).

Sont ainsi privilégiées des activités aux contours poreux, qui n'établissent pas de clôture entre un en-dedans et un en-dehors du Téléthon mais qui encouragent au contraire le passage du public d'une manifestation à une autre. La charge intentionnelle de l'engagement dans le Téléthon se voit également considérablement allégée au regard des exigences qu'appellent d'autres types de mobilisation collective puisque la forme même des activités qui y ont cours interdit toute sélection à l'entrée entre ceux qui adhèrent fortement à la cause du Téléthon et ceux qui entretiennent à son égard un lien beaucoup plus lâche : invitées à justifier leur présence dans l'arène des manifestations, les personnes avancent ainsi des raisons hétérogènes ( « c'est pour les enfants malades », « pour les handicapés ») qui contrastent avec les objectifs officiels assignés à la mobilisation.

\section{Le comptage des liens}

La collecte d'argent supposant une intense activité de solidarisation et de communalisation, tout, dans le Téléthon est prétexte à mobilisation, à la condition que soit favorisée la coopération mutuelle. Que les actions soient absurdes ou grotesques ou qu'elles donnent lieu à la production d'objets monstrueux et éphémères importe finalement peu. L'importani réside dans la manière dont elles tendent à faire apparaître aux personnes leurs facultés communes à agir de façon solidaire, à faire circuler entre elles des " positivités " à faire émerger en elles des potentialités qu'elles ne soupçonnent peut-être pas - une inclination altruiste, une capacité à agir pour les autres, un souci moral - à puiser en elles, comme le dit le slogan de l'AFM, « le meilleur de soi-même ». Une typologie un peu rudimentaire articulée autour des types de médiations permet ici de distinguer sept catégories d'actions : celles qui appellent une mise en mouvement du corps sans passer par l'entremise d'un objet (marche, course, nage, mime, comédie, etc.), celles qui réclament le recours à un objet particulier (tir à l'arc, football, scrabble, sculpture sur glace, pliage de cocottes en papier, dressage de chiens, pilotage de montgolfière), celles qui peuvent être regardées comme des formes allégoriques des liens de solidarité qui unissent les acteurs du Téléthon (chaînes humaines, cercles, rondes, feux de 
joie, etc.), celles qui sont articulées autour de dispositifs métrologiques catalyseurs d'énergie que l'on peut qualifier d' "agitomètres ", c'est-àdire des choses utilisées à la fois comme support de l'action et comme toise de la dépense d'énergie (la grande échelle des pompiers sans cesse montée et descendue), celles qui produisent des objets ubuesques et éphémères (pull de 9 mètres d'envergure, omelette de 100 œufs, boudin le plus long du monde, etc.), lourds des personnes qui les ont engendrés, car ils supposent la coopération d'un grand nombre d'acteurs autour d'eux (tant pour leur confection que pour leur absorption), et celles qui s'organisent autour de la vente d'objets marchands (vente de sucettes le long d'un relais pédestre, vente de pâtés en croûte). À l'exception de cette dernière catégorie, toutes les autres actions de la « Force $T$ » ont pour particularité de produire des biens non marchands. Ils n'ont de valeur que le temps que dure le Téléthon. Dès que celui-ci prend fin, ils perdent toute utilité car ils ne sont plus animés. C'est bien en ce sens que l'on peut dire qu'ils servent à manifester l'existence de la communauté agissante du Téléthon.

Le dispositif de collecte proprement dit n'est jamais totalement disjoint des actions collectives organisées en public. Il ne vient qu'exceptionnellement se greffer, tel un appendice extérieur, sur les rassemblements auxquels le Téléthon donne lieu et s'incarne alors dans des réceptacles fixes (comme les troncs d'églises) ou ambulants (comme ces boîtes tintantes de pièces que tendent les bénévoles d'associations caritatives). Il est le plus souvent enfermé dans le cours même de la mobilisation : chaque dispositif de connexion est en effet associé à un ou plusieurs outils de comptage et de conversion monétaire, qui autorisent la traduction en argent des actions communes : "Soixante perchistes se sont mobilisés depuis hier, 18 heures, sur la place de J. pour tenter de sauter plus haut que le Puy-de-Dôme, soit 1465 mètres, en sauts cumulés bien sûr " se réjouit un animateur. "Ils ont très largement dépassé leur défi avec un total de 2369 mètres en 705 sauts. Dix francs ont été récoltés pour chacun des sauts réalisés dans le cadre de ce défi » (Téléthon 96). Le dispositif de collecte se voit ici incorporé dans le cours des actions auxquelles tout un chacun est invité à participer : les cyclistes, les coureurs, les danseurs, etc. ne pédalent. courent, dansent, etc., que si leurs mouvements ont été payés par d'autres ou par eux-mêmes. Chacun, dans de telles circonstances, endosse à la fois l'habit du donateur et du collecteur.

Après avoir fait l'objet d'un découpage en unités ad hoc et avoir été mesurée à l'aune du même étalon, chacune des actions de la « Force $T$ » est vendue et achetée. Il cst alors possible d'agréger le montant des différentes manifestations se déroulant dans la même aire géographique. Avant de parvenir au compteur, la somme ainsi récoltée transite depuis le PC départemental jusqu'au PC central en empruntant les différents 
maillons de la chaîne mise en place par l'AFM. Ainsi, la valeur monétaire du chiffre qui circule vers le compteur télévisé est toujours animée du mouvement que lui ont imprimé le pliage de cocottes en papier, les mouvements de brasse des nageurs, les coups de rames des avironneurs ou le tricotage des grands-mères d'une association de quartier. Le chiffre qu'exhibe le compteur ne renvoie pas à un dénombrement des individus qui se sont mobilisés à l'occasion du Téléthon - et dont l'association pourrait se réclamer, à l'instar des hommes politiques s'efforçant de faire exister « leur » électorat en sommant les suffrages s'étant portés sur leur nom (6) -, mais bien à la traduction monétaire des mouvements qu'ils ont accomplis. Rien d'étonnant donc à ce que le compteur soit toujours animé puisque qu'il renvoie moins à un état ou une qualité (être un " donatcur » du Téléthon. être " généreux ») qu'à un processus (l'action concertée, la solidarisation, la communalisation). Le compteur contribue ainsi à fixer une certaine représentation de la réalité de la « Force $T$ » qu'il est censé, en partie. refléter. Il n'en constitue pas pour autant un artefact. Car le collectif qu'il contribue à faire exister et à représenter par une somme d'argent, renvoie à un groupe effectivement mobilisé et non à une collection artificielle d'individus atomisés. Les outils de communication à distance (télévision, radio, téléphone), en rendant sensible la simultanéité des mobilisations, en contribuant tout autant à fortifier les cercles primaires (de voisinage, de corps de métier, etc.) qu'à exacerber la ferveur nationale, contribuent indéniablement, bien qu'ils lient des individus distants les uns des autres, à la construction de ce sentiment d'appartenance communautaire. Comme le dit Durkheim (1912:598) : "la société ne peut faire sentir son influence que si elle est un acte, et elle n'est un acte que si les individus qui la composent sont assemblés et agissent en commun. C'est par l'action commune qu'elle prend conscience de soi et se pose; elle est avant tout une coopération active. Même les idées et les sentiments collectifs ne sont possibles que grâce à des mouvements extérieurs qui les symbolisent».

Le compteur renvoie ainsi à deux réalités fondamentalement différentes : il correspond bien à une addition d'argent, une somme des dons collectés ; mais il représente surtout des variations, un accroissement constant de cette somme. Ainsi, le compteur, dans sa première dimension, représente de fait la sommation de gestes individualisables, discrets au sens des statisticiens (tel individu a promis telle somme, les bordereaux de

(6) Sur le travail d'agrégation, par les professionnels de la politique, d'opinions dissemblables en " électorats " enfermant une conception égalitariste et universaliste des opinions, voir Offerlé (1988). 
promesses de don portent la trace de cet acte). Mais, il représente aussi plus que cette simple somme : dans l'expansion du chiffre dont il est sans cesse, et jusqu'aux dernières minutes de l'émission, l'index, il signifie le sens de la représentation télévisuelle, faite d'allers et retours entre scène centrale et scènes périphériques, de rencontres entre acteurs issus de divers horizons, d'un rassemblement progressif et convergent des mobilisés. Il montre ainsi la dynamique de constitution d'une communauté solidaire ; il l'établit, la fait exister. Cette dynamique, tout comme sa mise en scène pointe vers un lieu, le plateau central où est placé le compteur, et un moment : celui où son chiffre dépasse sa référence " réaliste ", celui de l'année précédente. On est samedi soir, il est presque deux heures du matin, heure à laquelle l'émission s'achève (Téléthon 96, samedi, 01:51):

Claude Sérillon : "Merci beaucoup Philippe! On va refaire un point compteur avec Jean-Pierre Spiero toujours en direct de l'Agora d'Evry!».

Michel Drucker : «C'est un moment important»,

Gérard Holtz : "Une heure cinquante »,

Claude Sérillon : "Pour ce dixième Téléthon! On est à 375000 ! Regardez bien! 378000 millions! Record battu! 378460994 francs ! 378 millions!" .

Musique, Applaudissements.

Michel Drucker :«Record battu!",

Gérard Holtz: « Le parrain! »,

Claude Sérillon : "Il y aura encore plus d'argent que l'année dernière ",

Michel Drucker :«Et ce n'est pas encore terminé ! »,

Claude Sérillon : "C'est un signe de confiance très fort que vous donnez! Continuez!", puis, en chantant : "Ce soir on vous met le feu ! Ce soir on vous met, ce soir on vous met le feu! Ce soir on vous met, on vous met le feu!».

Applaudissements. L'orchestre reprend la chanson des animateurs. Tout le monde crie. L'orchestre s'arrête. Applaudissements.

Gérard Holtz : "Ouha ouh! C'est génial!".

Claude Sérillon et Gérard Holtz (reprenant la chanson accompagnés par l'orchestre) : "Ce soir on vous met, ce soir on vous met, on vous met le feu! ».

Lorsqu'ils s'arrêtent, cris et applaudissements.

Gérard Holtz : "Bravo! (...) Thiern;, vous êtes le parrain d'une somme qu'on n'avait jamais obtenue encore dans le Téléthon »,

Thierry Lhermitte : "Et c'est pas fini alors continuons encore un petit peu. Allez! encore un petit peu! 36.37 ou alors 36.15 », 
Claude Sérillon: "Si quelqu'un a envie de se coucher tôt, qu'il nous le dise hein!",,

Thicrry Lhermitte : "C'est formidable! »,

Claude Sérillon: " $I l$ y a des voitures à pédale qui arrivent maintenant. Je crois qu'ils ont battu un record",

Gérard Holtz: "Ils ont fait une centaine de kilomètres!" .

La première caractéristique de cet événement est qu'il fonctionne d'abord comme une preuve du caractère imprévisible de la mobilisation. Le compteur loge, en quelque sorte, à la même enseigne l'ensemble des participants du Téléthon : personine ne sait précisément où il s'arrêtera ; quiconque, quidam comme organisateur, donateur, simple spectateur, ou lecteur des journaux du lendemain, présentatcur du Téléthon, ou président de l'AFM, est surpris du chiffre qu'il finit par afficher. Ce chiffre final du compteur ne se donne pas ainsi à voir comme le résultat d'une processus mécanique, comme la simple application d'une recette par quelque spécialiste de marketing. Le compteur apparaît comme une instance tierce, dotée d'une extériorité simultanée à tous, animateurs, producteurs, téléspectateurs ou malades. Au-delà de son imprévisibilité, c'est le dépassement lui-même qui est applaudi. C'est lui qui importe, bien plus que le chiffre final du compteur - qui en vient parfois à passer presque inaperçu, comme ce fut le cas lors du Téléthon 1996 (7). L'intérêt se focalise moins sur le chiffre du compteur, sur l'addition des sommes d'argent - dont le calcul ne deviendra définitif qu'une fois sorti de l'espace du Téléthon - que sur l'autre dimension qu'il désigne et qui est plus que sa simple somme : le processus même de mobilisation et de rassemblement des hommes. Ainsi, les variations du compteur ne représentent pas la communauté, mais concentrent l'attention du public sur le geste exemplaire de son rassemblement et de sa solidarisation. En ce sens, il institue bien la communauté telle qu'elle doit être. Le compteur, en ces instants, n'est plus la mesure de l'argent récolté, ni l'instrument performatif de l'engagement, il s'apparente au législateur du contrat social du Téléthon (8).

(7) Les deux chiffres étant relativement proches ( 378 millions pour 1995, 384 millions pour 1996) le second ne pouvait à lui seul incarner l'idée du record. Il en eut certainement été autrement si les 400 millions avaient été atteints.

(8) On se souvient que, dans la construction rousseauiste du Contrat social, le législateur intervient pour remédier aux errements éventuels du peuple. C'est lui qui, médiatisant la volonté générale avec elle-même, « constitue la République » et propose sans imposer les lois. Cette possibilité lui est ouverte pour la seule raison qu'il est doté d'une extériorité à la société. Il est une instance tierce, simultanément extérieure à tous les contractants et en opposition aux intérêts de chacun. 


\section{RÉFÉRENCES BIBLIOGRAPHIQUES}

Anderson B., 1996, L'imaginaire national. Réflexions sur l'origine et l'essor du nationalisme, Paris, La Découverte (1 ère édition américaine, 1983).

Boltanski L., 1993, La souffrance à distance. Morale humanitaire, médias et politique, Paris, Métailié.

Bruckner P., 1995, La tentation de l'innocence, Paris, Grasset.

Cardon D., 1995, “Chère Ménie... », Émotions et engagements de l'auditeur de Ménie Grégoire, Réseaux, 70, mars-avril, 41-78.

Chalvon-Demersay S., Rosental P.A., 1998, Une démographie des comportements volatils ? L'émergence de la micro-analyse dans la mesure de l'audience, Quaderni, 35, 129-147.

Dayan D., Katz E., 1996, La télévision cérémonielle. Anthropologie et histoire en direct, Paris, PUF (1 ère édition américaine, 1992).

Durkheim E., 1912 (édition de 1985), Les formes élémentaires de la vie religieuse. Le système totémique en Australie, Paris, PUF.

Favret-Saada J., 1977, Les mots, la mort, les sorts, Paris, Gallimard.

Hennion A., Méadel C., 1988, Radios, réseaux, régies. La représentation du public par les professionnels de la radio, rapport pour le GRECO Communication, Paris. Centre de sociologie de l'innovation, octobre.

Liebes T., Katz E., 1993, Six interprétations de la série « Dallas ", Hermès, $11-12,125-144$.

Mannoni O., 1969, Clefs pour l'imaginaire, Paris, Le Seuil.

Mattelart A., Neveu E., 1996, Cultural studies stories. La domestication d'une pensée sauvage, Réseaux, 80, novembre-décembre, 11-58.

Mauss M., 1950, Essai sur le don, forme et raisons de l'échange dans les sociétés archaïques, Sociologie et anthropologie, Paris, PUF, 145-279.

Meyrowitz J., 1985, No sense of place the impact of electronic media on social behavior, Oxford, Oxford University Press.

Offerlé M., 1988, Le nombre des voix. Électeurs, partis, et électorat socialistes à la fin du $\mathrm{XIX}^{\mathfrak{e}}$ siècle en France, Actes de la Recherche en Sciences Sociales, 71-72, 5-21.

Pasquier D., 1995, "Chère Hélène ». Les usages sociaux des séries collège, Réseaux, 70, mars-avril, 9-39.

Pharabod A.S., 1993, Perdu de vue, ethnographie d'un realiti-show, « Montrer, ne pas montrer », Cinéma, Rites et Mythes Contemporains, 16, 145-297. 


\section{Who makes shift the pledge meter of the French Telethon. A televisual construction of solidarity}

Since December of 1987, the Association francaise de lutte contre les myopathies (AFM) (the French Muscular Distrophy Association), organizes the French Telethon, in order to support scientific research and to help the victims of muscular diseases. Unlike most television programs, the Telethon is a unique apparatus which aim is to provoke solidarity, and as such is quite exemplary of the transformations that affect the televisual public sphere, the forms of the solidarity and the research public policies. This article deals with the role played by the pledge meter of the French Telethon, and analyses the conditions of emergence, during the show, of a bound up community. It tackles successively the television show, the reception of the program and the local events organized during the television program. The final figure of the meter can not be surely linked to the evolution, measured in real time, of the solidarity events; nor to the strategies of the producers of the program. That is by the variations of the figure, translating in accelerations and decelerations the different deals (monetary and human), which ties every one and all to the courage of the sick persons and to the efforts of the scientists, that the meter device manage to come into sight all together the monetary value of the pledges and the solidarity between the people who make common cause in the Telethon community. 


\section{RESUMEN}

\section{Pero quién mueve el medidor del Téléthon ? La construcción televisual de la solidaridad}

Iniciado en 1987 por la Asociación Francesa contra las Miopatías (AFM) para recoger fondos con el fin de apoyar la investigación científica y la ayuda a los enfermos, el Télethon constituye un dispositivo de solidaridad ejemplar de las transformaciones que afectan al mismo tiempo al espacio público televisual, al mundo de la solidaridad asociativa y a las políticas de orientación y de financiamiento de la investigación clínica. Este artículo, consagrado al papel que juega el medidor de promesas de donación del Téléthon, propone un análisis de las condiciones que encabezan la emergencia, durante la emisión, de una comunidad solidaria. Aborda sucesivamente la representación televisiva, la recepción del programa y todos los tipos de acciones locales que acompañan a la emisión. La evolución del medidor no puede ser solamente atribuida a las intenciones estratégicas de los programadores ni tampoco únicamente a la tarea de recuento estadístico del dinero recaudado. Es por su movimiento mismo, que traduce bajo la forma de aceleraciones o desaceleraciones las diferentes transacciones (monetarias y humanas) que ligan a todos y a cada uno con el valor de los enfermos y con los esfuerzos de los investigadores, que el dispositivo de recuento logra poner de relieve al mismo tiempo el valor monetario de las promesas de donación y la solidaridad entre las personas que se reconocen en la comunidad del Téléthon. 